\title{
Why not simply treat large adenomas of the rectum and ileal pouch after colectomy in familial adenomatous polyposis?
}

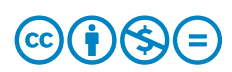

\author{
Authors \\ Institutions \\ 1 Gastroenterology Unit, E. Herriot Hospital. Hospices \\ Civils de Lyon, Lyon, France \\ 2 Digestive Surgery, E. Herriot Hospital, Hospices Civils de \\ Lyon, Lyon, France \\ Bibliography \\ DOI https://doi.org/10.1055/a-0890-3379 | \\ Endoscopy International Open 2019; 07: E699-E700 \\ (c) Georg Thieme Verlag KG Stuttgart · New York \\ elSSN 2196-9736
}

Jean-Christophe Saurin ${ }^{1}$, Nicolas Benech ${ }^{1}$, Laura Calavas ${ }^{1}$, Arnaud Pasquer $^{2}$

\author{
Corresponding author \\ Jean-Christophe Saurin, Gastroenterology unit, Pavillon L $2^{\circ}$ \\ et., Hôpital E. Herriot, 5 PI d’Arsonval, 69437 Lyon Cedex 03 \\ Fax: +33472110147 \\ Jean-christophe.saurin@chu-lyon.fr
}

Familial adenomatous polyposis (FAP) is a dominant or recessive genetic predisposition to colorectal cancer through development of hundreds or thousands of polyps in the colorectum. The APC (adenomatous polyposis coli) gene-driven form is associated with a $100 \%$ lifetime risk of colorectal cancer so that patients almost all require colectomy (with preservation of about $15 \mathrm{~cm}$ of rectum) or proctocolectomy (with the construction of an ileal pouch and ileo-anal anastomosis. The natural history of the rectal stump after colectomy is known from old series, as development and progression of rectal adenomas is systematic: the cancer risk is between $10 \%$ and $37 \%$ of cases 20 years after surgery [1]. The natural history of the ileal pouch is also relatively well known: $36 \%$ of patients develop new adenomas in the ileal stump after a mean of 7 years, including high-grade dysplasia [2]. Adenocarcinomas in the ileal pouch represent a well-documented situation, and may appear directly within the ileal mucosa, although some of them may begin within remaining rectal mucosa at the anastomosis [3].

This interesting series from Tajika et al shows the long-term outcome of systematic surveillance of the rectal stump in 14 patients and of the ileal pouch in 24 patients with FAP [4]. Median follow-up is 21 years, which allows interesting input regarding the natural history of adenomas. The result is not unexpected knowing the high incidence of adenoma development in this situation, but still important: 6 patients $(23 \%)$ developed adenocarcinoma (2) or advanced adenoma (4) in the ileal pouch a mean of 11 years after surgery. The small group of patients with an ileorectal anastomosis in this series present with a very benign evolution (although all present with adenomas during the surveillance), with no case of advanced adenoma and no case of cancer, but a very short follow-up of 2.1 years after surgery. The authors focus their discussion largely on risk of ileal pouch advanced neoplasia and cancer in the literature, which confirms: 1 . the significant risk of cancer in the ileal pouch with 22 cases reported; and 2 . the possibility of rapid development, in some cases less than 1 year after a "normal" endoscopy.

This series and the previous literature suggest important questions for clinical management of FAP:

- Is our surveillance adapted to early detection of advanced adenomas and cancer in FAP patients?

- Is our management/treatment adapted to prevention of advanced adenoma and cancer development?

About surveillance, why do cancers appear in patients less than 1 year after follow-up? One explanation could be an accelerated adenoma-carcinoma sequence. This accelerated carcinogenesis is the cornerstone of surveillance in Lynch syndrome, but has never been demonstrated in FAP, considering the evidence that adenomas are present during infancy with a median age of cancer at 38 [5]. One more logical (but unproven) explanation could be that we miss advanced neoplasia because of suboptimal examination of the rectum stump/ileal pouch. Interestingly, although there is a consensus for postsurgical surveillance after colectomy in FAP, every year, in the more recent recommendations [6], no methodological recommendation exists on the quality of this surveillance: no guideline on preparation, no guideline on dye-chromoscopy, for example. However, despite colectomy, patients with FAP can be difficult to prepare. Incomplete preparation probably should be an indication for repeated endoscopy, which has never been recommended. There may also be a role for optimized endoscopy using dye-chromoscopy. 
Our experience in FAP argues in favor of systematic indigo-carmine dye for polyp detection, especially in the ileal pouch where neoplastic lesions are almost systematically flat and poorly visible before chromoscopy [7]. However, as dye has been shown to improve detection of duodenal adenomas in FAP, no scientific evidence has been published in pouch and rectum [8]. Of course, a clear prospective demonstration of the importance of chromoscopy for examining the rectal/ileal pouch in patients with FAP would be of much higher value than this simple advice from an expert center.

About treatment, why don't national and international guidelines consider early treatment of rectal or ileal pouch adenomas in a situation where the cumulative risk of cancer is up to $37 \%$ at 20 years [6]? Would we leave a $10-\mathrm{mm}$ adenoma in place in a situation of average-risk colorectal cancer screening? Why do we leave in place large adenomas in patients with FAP during screening? Why do guidelines never recommend resection, at least, of large adenomas although we know that about $60 \%$ of $10-\mathrm{mm}$ adenomas contain foci of high-grade dysplasia or carcinoma $[9,10]$ ? The literature shows, at least in duodenal adenomas, that biopsies underestimate risk of high-grade dysplasia and cancer, and that almost all duodenal cancers in FAP arise from large, non-resected, adenomas $[11,12]$. Despite the very limited data and complete lack of randomized studies, there is some reported long-term experience with endoscopic "prophylactic" treatment in the rectal stump and ileal pouch in patients with FAP that suggests the feasibility of modifying this frightening and probably quite frequent evolution into advanced adenoma and cancer [13].

In 2019, we clearly don't have sufficient evidence-based data to recommend systematic treatment of large ( $>5 \mathrm{~mm}$ ? $>10 \mathrm{~mm}$ ?) ileal pouch or rectal adenomas in patients with FAP. But we have a lot of literature and clinical evidence of (longterm) evolution into advanced adenomas and cancer in both situations. Treatments like APC coagulation and resection are very common and relatively simple. We definitely need prospective studies to better evaluate the efficacy of systematic adenoma treatment in prevention of rectum/pouch cancer and secondary surgery. However, this may be long and difficult in this situation of rare disease. In the meantime, perhaps experts should consider reasonable "practice guidelines," including quality diagnostic endoscopy and some more aggressive therapeutic preventive approaches in patients with FAP.
Competing interests

None

References

[1] Church J, Burke C, McGannon E et al. Risk of rectal cancer in patients after colectomy and ileorectal anastomosis for familial adenomatous polyposis: a function of available surgical options. Dis Colon Rectum 2003; 46: $1175-1181$

[2] Church J. Ileoanal pouch neoplasia in familial adenomatous polyposis: an underestimated threat. Dis Colon Rectum 2005; 48: 1708-1713

[3] Palkar VM, deSouza L], Jagannath P et al. Adenocarcinoma arising in "J" pouch after total proctocolectomy for familial polyposis coli. Indian J Cancer 1997; 34: 16-19

[4] Tajika M, Tanaka T, Ishihara M et al. Long-term outcomes of metachronous neoplasms in the ileal pouch and rectum after surgical treatment in patients with familial adenomatous polyposis. Endosc Int Open 2019; 07: E691 -E699

[5] Arvanitis ML, Jagelman DG, Fazio VW et al. Mortality in patients with familial adenomatous polyposis. Dis Colon Rectum 1990; 33: 639642

[6] Syngal S, Brand RE, Church JM et al. ACG clinical guideline: Genetic testing and management of hereditary gastrointestinal cancer syndromes. Am J Gastroenterol 2015; 110: 223 -262; quiz 263

[7] Slim R, Ponchon T, Chavaillon A et al. Follow-up and ILaser Nd-YAG treatment of adenomas in the rectal stump of familial adenomatous polyposis (FAP) patients: long term results. Gastroenterology 2000: Abstr 118: 101153

[8] Dekker E, Boparai KS, Poley JW et al. High resolution endoscopy and the additional value of chromoendoscopy in the evaluation of duodenal adenomatosis in patients with familial adenomatous polyposis. Endoscopy 2009; 41: 666 - 669

[9] Lepilliez V, Chemaly M, Ponchon T et al. Endoscopic resection of sporadic duodenal adenomas: an efficient technique with a substantial risk of delayed bleeding. Endoscopy 2008; 40: 806-810

[10] Saurin JC, Ligneau B, Ponchon T et al. The influence of mutation site and age on the severity of duodenal polyposis in patients with familial adenomatous polyposis. Gastrointest Endosc 2002; 55: 342 - 347

[11] Groves C], Saunders BP, Spigelman AD et al. Duodenal cancer in patients with familial adenomatous polyposis (FAP): results of a 10 year prospective study. Gut 2002; 50: 636-641

[12] Napoleon B, Gincul R, Ponchon T et al. Endoscopic papillectomy for early ampullary tumors: long-term results from a large multicenter prospective study. Endoscopy 2014; 46: 127-134

[13] Moussata D, Nancey S, Lapalus MG et al. Frequency and severity of ileal adenomas in familial adenomatous polyposis after colectomy. Endoscopy 2008; 40: 120-125 